\title{
A fuzzy-based Bayesian Belief Network approach for railway bridge condition monitoring and fault detection
}

\author{
Matteo Vagnoli, Rasa Remenyte-Prescott, John Andrews \\ Resilience Engineering Research Group, Faculty of Engineering, University of Nottingham, University Park
}

Nottingham, NG7 2RD

\begin{abstract}
More than 35\% of the European railway bridges are over 100 years old and the increasing traffic loads are pushing the railway infrastructure to its limits. Bridge condition-monitoring strategies can help the railway industry to improve safety, availability and reliability of the network. In this paper, a Bayesian Belief Network method for condition monitoring and fault detection of a truss steel railway bridge is proposed by relying on a fuzzy analytical hierarchy process of expert knowledge. The BBN method is proposed for obtaining the bridge health state and identifying the most degraded bridge elements. A Finite Element model is developed for simulating the bridge behaviour and studying a degradation mechanism. The proposed approach originally captures the interactions existing between the health state of different bridge elements and, furthermore, when the evidence about the displacement is introduced in the BBN, the health state of the bridge is updated.
\end{abstract}

\section{INTRODUCTION}

Bridges are a vital element of the railway network. Across Europe there are more than 300,000 railway bridges for 212,000 km of railway (European Commission, 2012). More than $35 \%$ of these bridges are over 100 years old and, thus, the continuous exposure to various environmental conditions, such as earthquakes, wind and traffic, can affect the safety and the whole life cost of these railway assets. For example, Network Rail, which is the owner of the railway network in the UK, estimates to spend 35 billion of pounds over a 5-year period for maintenance and renewal activities of the railway network, and Irish Rail, the owner of the Irish railway networks, estimates to spend 20 million of Euros per year only on renewals and maintenance activities of the railway bridges alone (Network Rail, 2014), (AECOM, 2011).

Structural Health Monitoring (SHM) methods are needed in order to accurately monitor and assess the health state of the bridge and of its elements, without relying to subjective and intermittent visual inspections. Indeed, SHM aims to point out changes in the properties of the bridge in terms of degradation of materials and geometry of the element, which can critically affect the safety and reliability of the bridge. Measured behaviour of the bridge, such as acceleration, displacements, mode shape, frequencies, etc. can be analysed. Visual inspections, however, examine the bridge structure by relying on the experience of SHM experts, and as a consequence, some faults might not be detected adequately (Wang et al., 2009).

Two main approaches to SHM are possible: model-based and non-model-based reasoning methods (Doebling et al., 1998). A model-based method detects faults by implementing a mathematical model of the bridge (such as a Finite Element (FE) model), which describes the physical laws of the structure behaviour using the systematic knowledge of the engineers who design bridges or develop bridge Finite Element (FE) models, in order to evaluate the structural parameters of the bridge. On the other hand, non-model-based methods derive behavioural models by analysing the health condition using the measured data directly, and the faults are detected relying on the signal processing of the measured experimental data. In this latter case, the models may not have any direct physical significance, as it is in the former case (Venkatasubramanian et al., 2003), (Sanayei et al., 2015). Many SHM studies on railway bridges have been developed by adopting either the model-based (Teughels et al., 2002), (Sanayei et al., 2015) and the non-model based (Zhong et al., 2014), (Kim et al., 2015) approaches. The influence of each bridge element (such as abutments, slabs, joints, girder, bearings, etc.) on the bridge health state is not usually considered. However, this influence is extremely important to find out cause-effect relationships among elements exposed to a degradation process.

The aim of this paper is to propose a Bayesian Belief Network (BBN) condition monitoring and 
fault detection method in order to monitor and assess the health state of the whole bridge, by innovatively taking account of the health state of each bridge elements at the same time.

The BBN is developed by taking into account the knowledge of several bridge engineers using a Fuzzy Analytical Hierarchy Process (FAHP) (Dağdeviren et al., 2008), (Kabir et al., 2016), where fuzzy relative importance functions are defined to describe the vagueness and subjectivity in expert judgment through a relationship between linguistic variables. These variables are used to investigate potential relationship between different bridge elements and numerical intervals, which numerically quantify the opinion of the expert. The performance of the BBN method is demonstrated by developing an FE model of a truss steel railway bridge. The unavoidable micro-cracks of the joints, which are difficult to spot during a visual inspection, are considered as the degradation mechanism. Finally, displacements of the bridge when the bridge is in different health condition, which are retrieved from the FE model, are used as evidence of the bridge behaviour and, thus, as the input to the BBN.

The paper is organized as follows: Section 2 describes the methodology, i.e. the FE model and the degradation mechanism considered, the development of the BBN and the FAHP methods; Section 3 shows how the BBN works for a case study of degradation of a bridge element; the conclusion and the remarks are discussed in Section 4.

\section{THE METHODOLOGY}

The aim of this paper is to propose an SHM method, which is able to detect the unexpected behaviour of a railway bridge in order to provide rapid information to bridge managers about the health state of the bridge. For these steps, an FE model of a steel truss railway bridge is developed in order to simulate the expected behaviour of the bridge due to external loads, such as train load, and furthermore, the effect of a degradation mechanism of each component on the bridge can be analysed. Therefore, any change in the health state of the bridge can be associated with the health state and the degradation mechanism of its elements.

Then, a BBN of the bridge is developed by including the chords of the bridge, where one node in the $\mathrm{BBN}$ is assigned to each chord. The prior CPTs of the BBN are defined by merging the information about the behaviour of the bridge, which is obtained by using the FE model, and the information retrieved from interviews with bridge managers and structural engineers. This way, the influence among the health state of each bridge elements is evaluated by numerical data and by expert knowledge. An FHAP is then adopted in order to compute the influence of the behaviour of each bridge element on the health state of the bridge elements, and, consequently, on the health state of the whole bridge.

The method finally illustrates that, as soon as new evidence of the bridge behaviour becomes available, it is used as an input to the BBN, and the health state of the bridge and of its elements is automatically updated, and thus the unexpected behaviour of the bridge, and its most degraded element(s), can be identified.

\subsection{The Finite Element model of the steel truss railway bridge}

Steel railway bridges have been chosen in this paper since their degradation mechanisms, such as corrosion and cracks, which are easier to asses than those of the concrete structure, can develop rapidly after they have started and, consequently, an early detection and management of such condition can be of great importance to bridge owners for reducing the risk of failure and the whole-life cycle cost of the bridge (Katipamula et al., 2005). For these reasons, an FE model of a steel truss bridge was developed in this paper, with the aim of analysing and predicting the bridge behaviour due to degraded states of elements.

A warren steel truss bridge has been selected and modelled using the structural analysis software SAP2000, as shown in Fig. 1. The example bridge is $30 \mathrm{~m}$ long, $7 \mathrm{~m}$ wide and $8 \mathrm{~m}$ high. The components of the bridge have been realized considering the S355 steel, as this steel is commonly used in Europe to build steel railway bridges (Pipinato et al., 2016). The reference system used in this paper is as follows: the side of the bridge at $y=0 \mathrm{~m}$, is called the right side of the bridge, whereas at $y=7 \mathrm{~m}$ it is called the left side of the bridge. As it is assumed that the bridge allows the transit of trains in two directions, two railway tracks have been modelled by following the most commonly used dimensions (Country Regional Network, 2012).

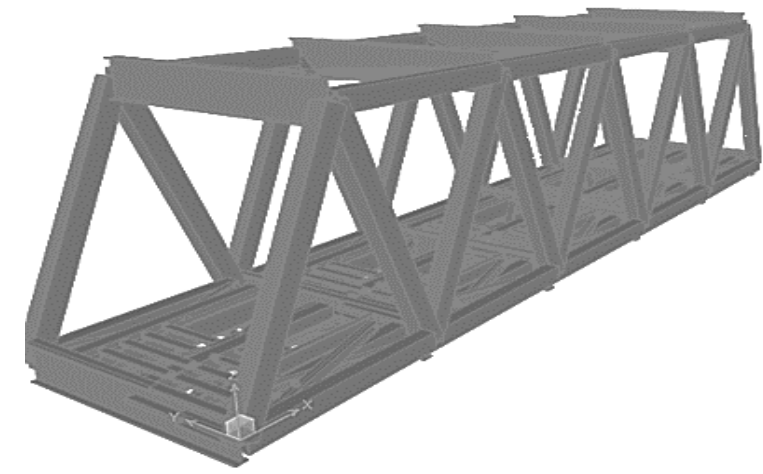

Figure 1 FE model of the steel truss railway bridge 


\subsection{The degradation mechanism}

The degradation mechanism of the developed steel truss bridge is analysed by considering the growth of micro-cracks at the joints (Mehrjoo et al., 2008). Micro-cracks, which are unavoidably created in the bridge elements during the welding and assembling phase of the bridge, are difficult to identify during a visual inspection, and more than $40 \%$ of the steel truss bridges are affected by this degradation mechanism. Furthermore, the bridge is continuously exposed to a cycle of loading and unloading, i.e. trains are continuously passing over the bridge, and, as a result, the size of the micro-cracks becomes larger. This degradation mechanism leads to a reduction of the cross sectional area at the joints which consequently suffers with fatigue. In order to simulate this degradation mechanism, in this study the cross sectional area of the degraded bridge elements has been reduced up to the $70 \%$ of its initial value.

As natural frequency and mode shape analysis have shown some limitations in SHM, in this paper displacements of the bridge joints are considered as the monitored parameter of the bridge behaviour. Indeed, frequency shifts caused by a failure can be small and thus they can be hidden due to the noise of the measurement; on the other hand, mode shape can be also prone to measurement contamination, and it is useful for SHM only if its measurement point is close to the node point for a particular mode (Doebling et al., 1998). Therefore, once a static uniform load of $40 \mathrm{kN} / \mathrm{m}$ has been applied to the bridge in order to simulate a train, which has been stopped on the track, the displacements at the joints are retrieved using the FE model.

Figure 2 shows the displacements of the top chord on the right hand side of the bridge, which have been calculated using the FE model when the bridge is in a healthy (solid line in Figure 2) and in a degraded state (the cross sectional area of the truss components is reduced by the $10 \%$ and the $30 \%$ of its initial value, as depicted in Figure 2 by the dotted and the dashed lines, respectively). The displacements of the degraded top chord are larger than those of the healthy case, and, moreover, as the bridge degradation grows, the displacements of the top chord on the right hand side of the bridge consequently increase.

\subsection{The Bayesian Belief Network}

In this paper, a BBN method is proposed in order to assess the health state of the whole bridge by monitoring the health state of its elements. BBN has been selected due to its ability to provide monitoring, diagnostic and predictive abilities, and furthermore, it allows an update of the probability distributions of the health states of the bridge and of its elements to

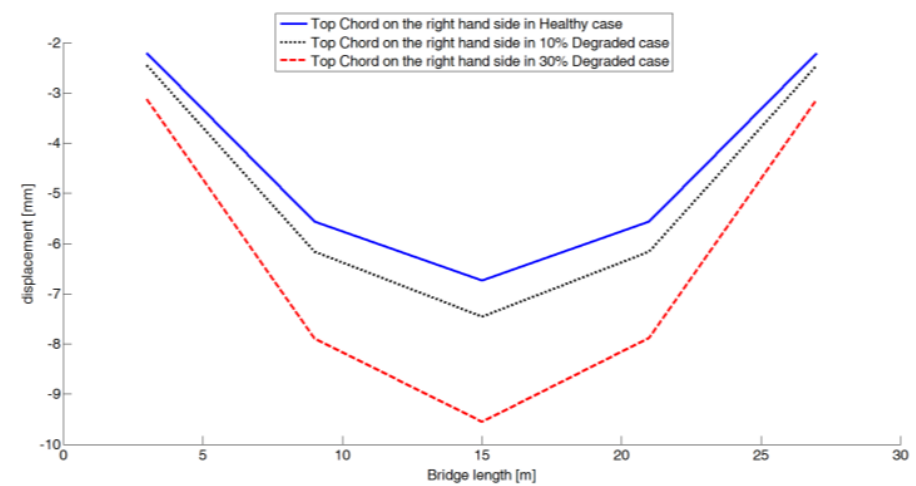

Figure 2 Displacements of the top chord on the right hand side of the bridge model

be calculated every time, when new evidence of the bridge behaviour is available.

The BBN of the steel truss bridge is developed by considering the bridge elements as nodes of the BBN: the 4 nodes on the highest layer of Figure 3 represent the virtual sensors of the FE model, which take as input the displacements of the steel truss bridge; 4 nodes, which are child node of the sensor nodes, belong to the middle layer in Figure 3. These nodes represent the major elements of the bridge (i.e. the top and bottom chords). Finally, at the bottom of Figure 3, the node called "Bridge Health State" represents the health state of the whole bridge.

It is worth mentioning that, as the BBN can describe the causes that influence a given effect by defying conditional dependencies among the variables, the sensor nodes, i.e. the 4 nodes on the top layer of Figure 3, are connected to each node representing the bridge elements. Indeed, once a degradation mechanism is initiated in an element of the bridge, and it is consequently measured by the sensors, other elements of the bridge are exposed to a higher load than before and, thus, their health state can be influenced.

The nodes representing the whole health state and the elements of the bridge, which are the nodes that have to be monitored with the aim of assessing their health states, have 3 mutually exclusive health states, which have been defined through expert elicitation (Rafiq et al., 2015): i) a healthy state, where the component of the truss bridge is in a good condition, and thus its displacements are equal to or within a $15 \%$ increase in displacements from the FE model of the healthy bridge; ii) a partially degraded state, where the increase in displacements is greater than $15 \%$ but less than $30 \%$; iii) a severely degraded state, if the increase in displacements of the bridge components is greater than $30 \%$.

In order to evaluate the dependencies among different elements of the bridge, conditional probabilities for each node are needed, given the health state of its parents in the network, describing the Conditional Probability Tables (CPT) for each node. The CPTs usually need exact information in the form of prior and conditional probabilities. However, exact values of dependencies are challenging to obtain due 
to high level of uncertainty that is usually present in the structural analysis of any civil infrastructure. Furthermore, bridge experts often provide uncertain answers rather than precise values, due to the fuzzy nature of the influence among different elements of the bridge (Torfi et al., 2010). For these reasons, a fuzzy linguistic approach is adopted in order to investigate the possible influence among the bridge elements by relying on the judgment of experts.

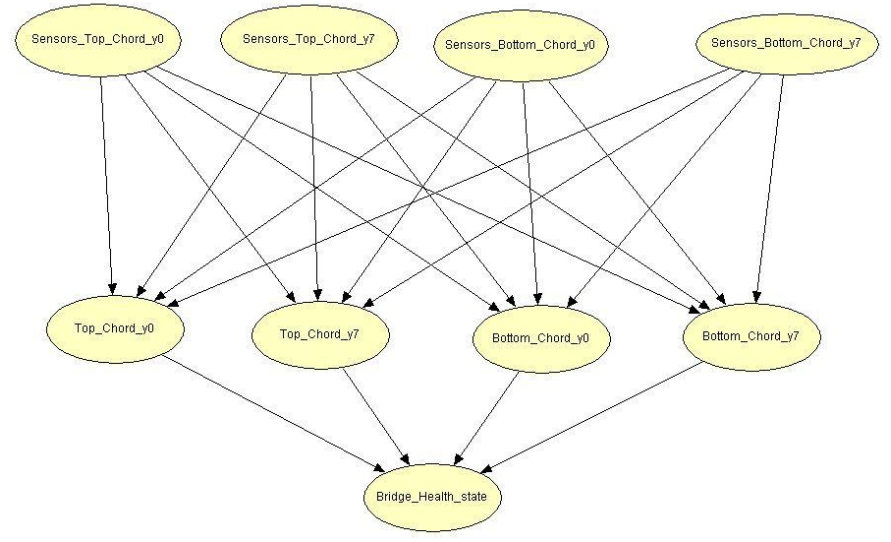

Figure 3 Bayesian Belief Network of the steel truss bridge

\subsubsection{Fuzzy Analytical Hierarchy Process}

In order to investigate how an element of the steel truss bridge is influenced by the degradation process of other bridge elements, a group of bridge experts has been interviewed. Particularly, the group of experts was composed of bridge engineers that are working in the private sector, as well as university academics that are teaching structural engineering courses. In this way, thanks to the heterogeneity of the interviewed group, both theoretical and practical expertise are considered in the analysis.

The experts have been called to answer a set of 11 questions, where a pairwise comparison between the degrading element of the bridge and other elements was carried out. As the experts are usually more confident to express their assessment by using language variables, instead of providing a crisp numerical value, the possible answers have been expressed as the probability using linguistic terms, as shown in Table 1. The linguistic fuzzy term gives the opportunity for the experts to include into their assessment the ambiguities, uncertainties, vagueness and incomplete information (Kulak et al., 2005). As soon as all experts have assessed the possible influences among the elements of the bridge, a triangular relative importance scale was assigned to their responses, as shown in Table 1 and Figure 4 (Dağdeviren et al., 2008). The different responses were then merged together by weighing the years of experience of the expert, i.e. the more experience the expert has, the greater the weight is assigned to his judgment (Kabir et al., 2016),

$$
W_{i}=\frac{E_{i}^{\beta}}{\max _{i=1}^{N}\left(E_{i}^{\beta}\right)}
$$

where $E_{i}$ is the number of years of experience of the expert $i ; \beta$ is a parameter for weight experts. The higher $\beta$, the more the judgment of the more experienced experts becomes dominant over other experts judgment.

Table 1 Linguistic probability scale and fuzzy membership function

\begin{tabular}{|c|c|c|}
\hline $\begin{array}{c}\text { Linguistic } \\
\text { probability } \\
\text { scale }\end{array}$ & $\begin{array}{l}\text { Triangular } \\
\text { fuzzy scale }\end{array}$ & Description \\
\hline $\begin{array}{l}\text { Very Unlikely } \\
\text { (VU) }\end{array}$ & {$[1 / 2,1,3 / 2]$} & $\begin{array}{l}\text { It is highly unlikely that influ- } \\
\text { ences occur }\end{array}$ \\
\hline Unlikely (U) & {$[1,3 / 2,2]$} & $\begin{array}{l}\text { It is unlikely but possible that in- } \\
\text { fluences occur }\end{array}$ \\
\hline $\begin{array}{l}\text { Even Chance } \\
\text { (EC) }\end{array}$ & {$[3 / 2,2,5 / 2]$} & $\begin{array}{c}\text { The occurrence likelihood of } \\
\text { possible influences is even } \\
\text { chance }\end{array}$ \\
\hline Likely (L) & {$[2,5 / 2,3]$} & It is likely that influences occur \\
\hline $\begin{array}{l}\text { Very Likely } \\
\text { (VL) }\end{array}$ & {$[5 / 2,3,7 / 2]$} & $\begin{array}{l}\text { It is highly likely that influences } \\
\text { occur }\end{array}$ \\
\hline
\end{tabular}

Hence, the optimal value of $\beta$ has been identified as 0.91 , by using a sensitivity analysis in order to avoid a single member judgment, i.e. the weight of the most senior expert is dominant over the weights of other experts, and to guarantee a group judgment based on the information retrieved by the whole group of experts. Therefore, the possible influence among the elements of the bridge has been assessed. For example, if 2 experts are considered, the first expert (Expert 1) with 3 years of experience, and the second expert (Expert 2) with 9 years of experience, Table 2 shows the responses to the following question: if a degradation mechanism is present on the Top Chord on the Right hand side (TRC) of the bridge, how likely are the other Bridge Elements (BE) to be influenced, such as Bottom Chord on the Right hand side (BRC), and Top and Bottom Chords on the Left hand side, (TLC) and (BLC) respective$l y$ ? Note that the weighted ensemble of the judgments of the experts is closer to the judgment of Expert 2 than to the one of Expert 1 due to the fact that Expert 2 is more experienced than Expert 1.

The FAHP is introduced to assess the weights of the unconditional probability of the parent nodes (i.e. the sensor nodes) on their child nodes (i.e. the nodes representing the top and bottom chords) in CPTs, by considering the ambiguity of human judgment. This analysis, which is carried out using the modified Chang extend analysis that has been demonstrated to perform better than the original Chang extend (Wang et al., 2006), leads to the evaluation of the importance weight vector of the fuzzy pairwise comparison matrix. The pairwise comparison matrix was obtained from the weighted judgements of the experts, by considering the pairwise comparison vector 
for the influence between each degraded element of the bridge and the other bridge elements.

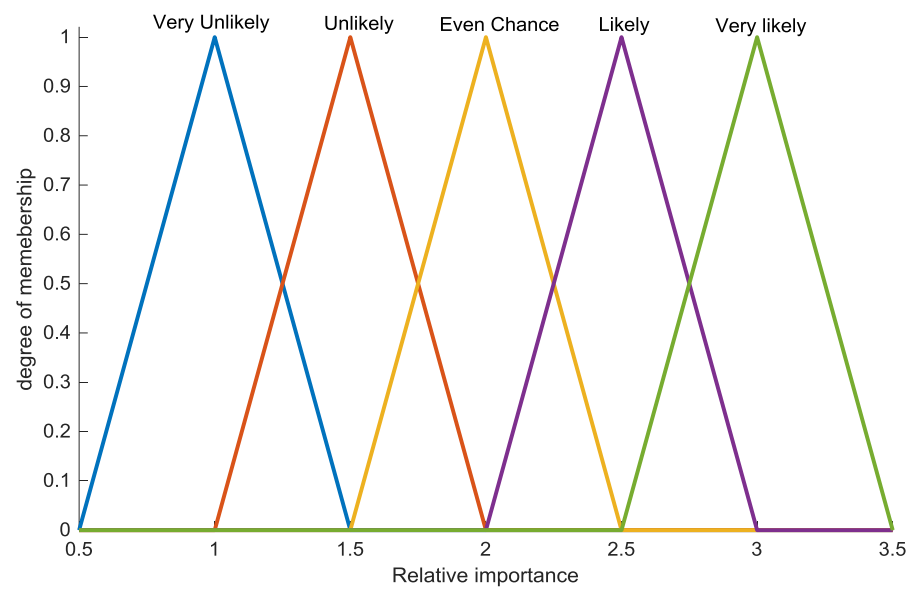

Figure 4 Linguistic scale for relative importance.

Table 2 Expert pairwise comparison between the degraded top chord on the right hand side and the others bridge elements

\begin{tabular}{|c|c|c|c|c|c|}
\hline & BE & TRC & BRC & TLC & BLC \\
\hline Expert 1 & TRC & {$\left[\begin{array}{lll}1 & 1 & 1\end{array}\right]$} & $\begin{array}{c}\mathrm{U} \\
{[1,3 / 2,2}\end{array}$ & $\begin{array}{c}\mathrm{U} \\
{[1,3 / 2,2]}\end{array}$ & $\begin{array}{c}\mathrm{VU} \\
{[1 / 2,1,3 / 2}\end{array}$ \\
\hline Expert 2 & TRC & {$\left[\begin{array}{lll}1 & 1 & 1\end{array}\right]$} & $\begin{array}{c}\mathrm{L} \\
{[2,5 / 2,3}\end{array}$ & $\begin{array}{c}\mathrm{L} \\
{[2,5 / 2,3}\end{array}$ & $\begin{array}{c}\mathrm{L} \\
{[2,5 / 2,3}\end{array}$ \\
\hline Weighted & TRC & {$\left[\begin{array}{lll}1 & 1 & 1\end{array}\right]$} & $\begin{array}{c}\mathrm{EC} \\
{[3 / 2,2,5 / 2}\end{array}$ & $\begin{array}{c}\mathrm{EC} \\
{[3 / 2,2,5 / 2}\end{array}$ & $\begin{array}{c}\mathrm{EC} \\
{[3 / 2,2,5 / 2}\end{array}$ \\
\hline
\end{tabular}

A detailed theoretical description of the FAHP and the Chang extend analysis are out the scope of this paper, and it can be found in literature (Wang et al., 2006), (Dağdeviren et al., 2008), (Torfi et al., 2010), (Kabir et al., 2016). The key steps of the FAHP are provided below.

Consider that a triangular fuzzy membership function is characterized by $\mu=(a, b, c)$, where $a<b<c$, and that $P_{j k}$ is the 1-pairwise comparison between the variable $j$ and $k$. The first step of the FAHP is to compute the fuzzy synthetic extent value, $S_{j}$ :

$$
S_{j}=\sum_{k=1}^{m} P_{j k} \otimes\left[\sum_{j=1}^{n} \sum_{k=1}^{m} P_{j k}\right]^{-1}
$$

where $n$ is the number of rows of the pairwise matrix, i.e., the number of variables for which a pairwise comparison is considered in the analysis, and $m$ is the number of columns of the pairwise comparison, i.e. the number of pairwise comparison for each variable considered. For example, Table 2 shows that the pairwise comparison is carried out for only one variable, $n=1$, and that this variable is evaluated in 4 pairwise comparisons, $m=4$. It is worth noting that $\otimes$ represents the extended multiplication for two fuzzy numbers.

The sum of the pairwise comparison of each variable (the first term of Eq. 2) can be easily computed as: $\sum_{k=1}^{m} P_{j k}=\left(\sum_{k=1}^{m} a_{k}, \sum_{k=1}^{m} b_{k}, \sum_{k=1}^{m} c_{k}\right)$

The second term of equation (2) is computed following the formula proposed by (Wang et al., 2006).

After computing the fuzzy synthetic extent value for each variable, the importance weight vector is computed by assessing the integral value of each synthetic extend value as follows:

$$
I_{j}=\frac{1}{2}\left(\alpha c_{j}+b_{j}+(1-\alpha) a_{j}\right)
$$

where $\alpha$ is the optimism of the expert, and $\left(a_{j}, b_{j}, c_{j}\right)$ is the fuzzy membership of the $j$-th fuzzy synthetic extent.

Finally, the importance weight vector of each variable can be assessed as:

$$
w_{j}=\frac{I_{j}}{\sum_{j=1}^{n} I_{j}}
$$

where $j=1,2, \ldots, n$.

It can be noted that the FAHP requires to verify the consistency of the pairwise comparisons. Indeed, the consistency ratio measures any inconsistency within the judgments in the comparison matrix. To do that, the fuzzy pairwise comparison matrix needs to be defuzzified, and, thus, in this paper, the defuzzification method of middle of maximum has been adopted in order to convert the fuzzy comparison matrix into a crisp matrix, which thereafter is used for the investigation of the consistency (Zhao et al., 1991). The consistency ratio results to be 0.0154 , and as it is lower than 0.1 , the inconsistency of the comparison matrix is satisfactory (i.e. the comparison matrix is consistent) and the experts do not need to revise their assessments (Dağdeviren et al., 2008).

The FAHP has been applied recursively in order to assess the importance weight vector of each node representing the health state of the bridge elements, i.e., the influence of the measured behaviour of each bridge element on the health state of bridge elements (the nodes that belong to the middle layer of Figure 3 ) is evaluated by using the FAHP. For example, Table 3 shows the importance weight vector for the influence of the behaviour of the TLC on the bridge elements: the sensors (or the modelled sensors using the FE model in the case study in Section 3) installed on the TLC measure the behaviour of this bridge element, and, consequently, the health state of all bridge elements is influenced by the following values of Table 3 . The values in Table 3 show that the health state of the element where the sensors are installed, the TLC in this case, is most influenced by sensor reading, whereas the farthest elements from the sensors, the BRC in this example, has the lowest weight and, thus, the lowest influence. 
Table 3 Importance weight vector for the influence of the TLC

\begin{tabular}{|c|c|c|c|c|}
\hline & TLC & BLC & TRC & BRC \\
\hline $\begin{array}{c}\text { Importance } \\
\text { Weight }\end{array}$ & 0.30 & 0.26 & 0.222 & 0.218 \\
\hline
\end{tabular}

\subsubsection{The CPT}

The values in the CPTs of the bridge health state and its elements are computed by defying the values for the prior probability distributions of the parent nodes and, then, the values are calculated by applying the importance weight vectors, retrieved using the FAHP of Section 2.3.1, and the results of the FE simulations, which have been performed in order to study the evolution of the micro-cracks degradation mechanism. Indeed, the FE simulations show that as the cross sectional area of the truss elements decreases, i.e. the dimension of the micro-cracks increases, the displacements of the top (bottom) chords increase, and, consequently, the health state of the component decreases (as shown in Figure 2). This is due to the relationship between the Young's modulus and the stresses.

Therefore, the process of CPTs definition starts by assigning the prior probability distribution to the 4 nodes representing the sensors, which take as the input the displacements of the steel truss bridge from the FE model. These nodes are arbitrarily defined by 6 possible states, by dividing the maximum difference between the displacements of the top (bottom) chords in the healthy and the most degraded case (i.e. when the cross sectional area of the degraded truss elements has decreased to the $70 \%$ of its initial value) into six equal steps (states from $\mathrm{A}$ to $\mathrm{F}$ in $\mathrm{Ta}-$ ble 4). The prior probability is defined by considering a $99 \%$ probability that the sensors of each chord are measuring the displacements for a healthy state, and, thus, the bridge and its elements are in the healthy state. Each health state (the healthy state, the partially degraded state, the severely degraded state) of the nodes representing the top and bottom chords, i.e. the nodes in the middle layer of Figure 3, has $1296=\left(6^{4}\right)$ possible ways to be influenced by their parent nodes, and this influence is governed by the importance weights vectors computed using Eq. 5, and the mathematical correlation between the degradation mechanisms and the bridge behaviour, which has been pointed out by studying several degradation levels of different elements of the bridge and analysing the corresponding bridge behaviour using the FE model. Therefore, the CPTs of the nodes of the top and bottom chords are composed by $3 \times 1296$ matrices, where the three rows are the three health states.

Table 4 shows that as the sensors installed on the top chords are measuring higher displacements, even if the sensors that are installed on the bottom chords are measuring displacements equal to those of the healthy FE model (state A, where the displacements are equal to the displacement of the healthy FE model), the health state of the bridge elements is conse- quently moving from the healthy state to the states, where the displacements are higher than those of the healthy FE model (states from B to F), and the probability that the top chord on the left-hand side is in the healthy state decreases, as shown by the bold values in Table 4.

\section{CASE STUDY}

As discussed in Section 2.2, in this paper the growth of micro-cracks at the joints location is considered as the degradation mechanism (Mehrjoo et al., 2008). The growth of micro-cracks is due to the continuous exposure of the bridge to a cycle of loading and unloading, i.e. trains continuously passing over the bridge. Figure 5 shows 7 evidence of the degradation mechanism of the TLC: as the micro-cracks of the joint of the top chord on the left hand side of the bridge become larger, the displacements of the TLC also increase. Indeed, the solid line of Figure 5 depicts the healthy state of the TLC, whereas, the nonsolid lines represent 6 degraded states of the TLC (i.e. the reduction of the cross sectional area of the truss members from the $5 \%$ up to the $30 \%$ of their initial values).

The displacements of the bridge elements are then used as the input to the BBN. In this way, the BBN can update the health state of the elements of the bridge, and consequently of the whole bridge, relying on displacement values. As new evidence becomes available, the probability of each health state for each node is updated, based on the evidence of the bridge behaviour. In terms of evidence about the gradual increasing of the size of the micro-cracks, as shown in Figure 5, the BBN updates the health state of the bridge, and of its elements, every time when a new measurement is available, as depicted in Figure 6 , where the posterior probability distributions of the health state of each node are updated, as soon as new data from the FE model is available, and it is used as the input to the BBN. Figure 6 shows that the probability of the partially degraded state of each bridge elements (node from 1 to 4 ) increases and,

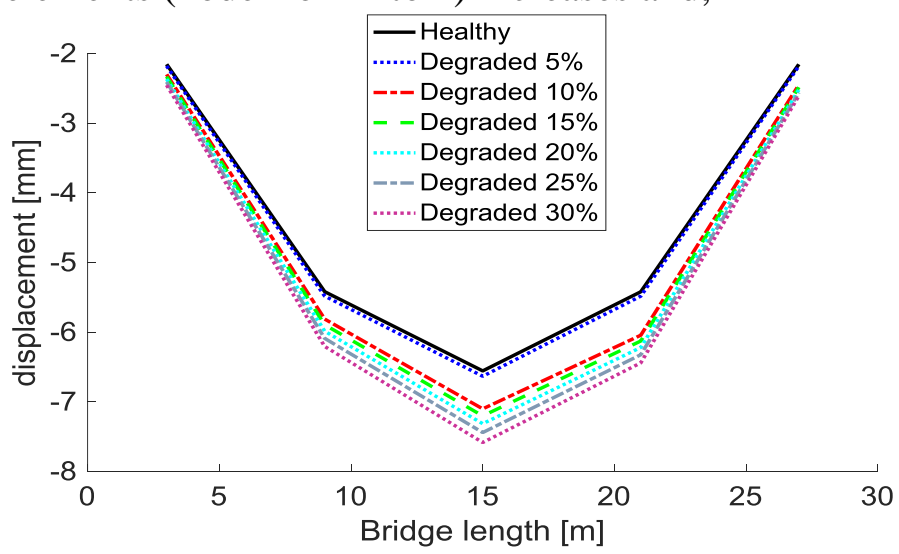

Figure 5 Displacements of the healthy and degraded top chord on the left hand side of the bridge model. 
Table 4 Extract of the CPT of the top chord on the left-hand side of the bridge (For brevity the part of CPT for states from $\mathrm{D}$ to $\mathrm{F}$ are omitted)

\begin{tabular}{|c|c|c|c|c|c|c|c|c|c|}
\hline \multicolumn{10}{|c|}{ Sensors of Bottom chords in state A } \\
\hline \multirow{2}{*}{\multicolumn{2}{|c|}{$\begin{array}{l}\text { Sensors at } \mathrm{y}=0 \\
\text { Sensors at } \mathrm{y}=7\end{array}$}} & \multicolumn{4}{|c|}{$\mathrm{A}$} & \multicolumn{4}{|c|}{$B$} \\
\hline & & $\mathrm{A}$ & B & $\mathrm{C}$ & \multirow{4}{*}{ From $\mathrm{D}$ to $\mathrm{F}$} & $\mathrm{A}$ & B & $\mathrm{C}$ & \multirow{4}{*}{ From $\mathrm{D}$ to $\mathrm{F}$} \\
\hline \multirow{3}{*}{$\begin{array}{l}\text { Health } \\
\text { State of the } \\
\text { TCL }\end{array}$} & $\begin{array}{c}\text { Healthy } \\
\text { state }\end{array}$ & 0.9 & 0.66 & 0.47 & & $\mathbf{0 . 8 3}$ & 0.54 & 0.35 & \\
\hline & $\begin{array}{l}\text { Partially } \\
\text { Degraded }\end{array}$ & 0.05 & 0.2 & 0.30 & & 0.1 & 0.26 & 0.37 & \\
\hline & $\begin{array}{l}\text { Severely } \\
\text { degraded }\end{array}$ & 0.05 & 0.14 & 0.23 & & 0.07 & 0.20 & 0.27 & \\
\hline
\end{tabular}

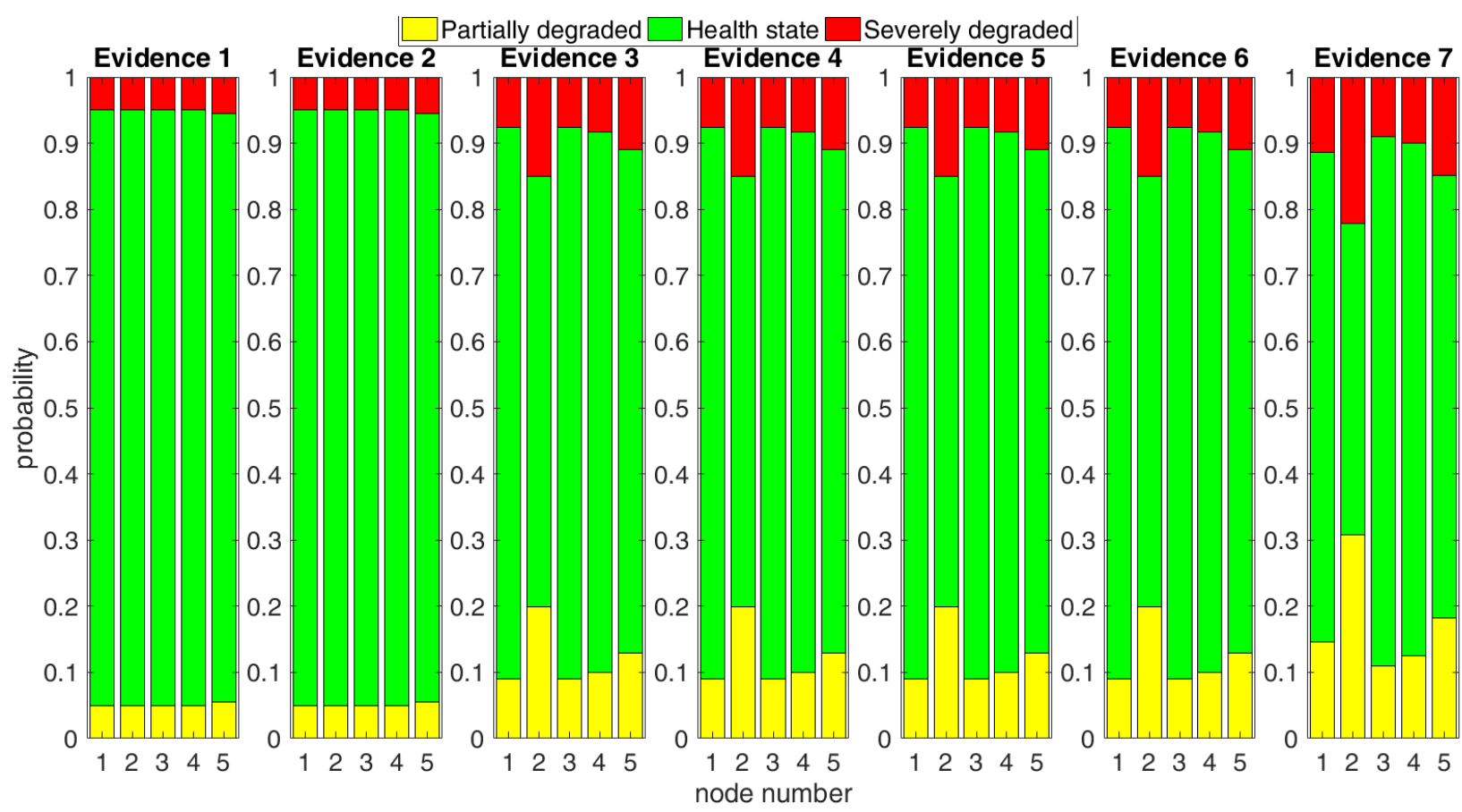

Figure 6 Posterior probability evolution using displacements as evidence of bridge behaviour.

consequently, the probability of the healthy state of the whole bridge (node 5) decreases. Particularly, the probabilities of the degraded states of the TLC (node 2) are subject to the highest increment, as the TLC is the degrading bridge element in this example. It is worth noting that following the importance weight vector of Table 3 , the BLC is the most influenced element by the degradation of the TLC and, consequently, its health states are updated by mainly increasing the probability of the partially degraded health state, as shown by node 4 in Figure 6 . Finally, the probability of the health states of the TRC and BRC, node 1 and 3 in Figure 6, respectively, are updated by nearly the same amount, as suggested by the FAHP.

Figure 6 shows that as soon as a new evidence of the bridge behaviour becomes available, in this case study the 7 displacement patterns provided by the FE model, the probability of each health state for each node is updated. Consequently, the probability of the whole health state of the bridge is monitored and the causes of the bridge deterioration are recognised. This way, the most degraded component of the bridge can be identified and the maintenance pro- gramme can be adequately scheduled, based on the degradation level of the bridge elements.

\section{CONCLUSION}

Although in the recent years the technology for data acquisition, processing and analysis has improved significantly, currently the bridge condition monitoring and fault detection is mainly carried out by adopting time-consuming and expensive visual inspections. Therefore, automatic SHM methods, which rely on the analysis of static and dynamic responses of the bridge in order to provide reliable assessment of the bridge health state, can drive bridge managers towards a better management of the asset, by assessing the health state of railway bridges reliably.

In this paper, an FE model of a truss railway bridge has been developed using the SAP2000 software. The unavoidable micro-cracks failures, which occur during the welding process of the joints in steel bridges, have been simulated by gradually reducing the cross sectional area of the truss elements of the bridge, in order to study this degradation 
mechanism of the bridge. A BBN has been developed by considering the bridge health state and its elements as the nodes of the BBN, and the knowledge of bridge structural experts on influence among different bridge elements has been considered. The developed BBN has demonstrated the ability to monitor the health state of the bridge and its components effectively, by updating their health state each time when new evidence of the bridge behaviour is available, and, consequently, the most degraded bridge component can be identified.

The proposed method is the first step towards meeting the requirements of a real time condition monitoring and fault detection tool of bridges, in order to achieve a reduction of the whole life cost of the asset by adopting a condition-based maintenance strategy. Indeed, a reduction of maintenance cost can be achieved by adequately planning the activities based on the health state of the elements of the bridge. Despite the fact that the method has shown good results in monitoring the health state of the bridge and of its components, several improvements are needed in order to achieve better accuracy of the fault detection method. For example, all possible dependencies between different elements of the bridge (such as the relationship between joints and beams within the chords) need to be considered in the structure of the BBN, and a more robust definition of the CPTs is needed. In addition, the proposed method could to be verified in a real framework, where sensors could be installed on a real bridge in order to collect data on bridge behaviour, and, thus, the bridge health state could be monitored in real time, to identify deteriorated bridge behaviour.

\section{ACKNOWLEDGMENTS}

This project has received funding from the European Union's Horizon 2020 research and innovation programme under the Marie Skłodowska-Curie grant agreement No. 642453.

The authors strongly thank all the experts for their availability and help.

\section{REFERENCES}

Aecom and Irish Rail, 2030 Rail Network Strategy Review Final Report, 2011.

Country Regional Network, Sleepers and track support, Engineering standard, track, 2012.

Dağdeviren, M., Yüksel, I., Developing a fuzzy analytic hierarchy process (AHP) model for behavior-based safety management, Information Sciences, 178 (6), pp. 1717-1733. 2008.

Doebling, S.W., Farrar, C.R., Prime, M.B., A summary review of vibration-based fault identification methods, the shock and vibration, Digest, 30 (2), pp. 91-105, 1998.
European Commission, EU transport in figures, statistical pocketbook, 2012.

Kabir, G., Sadiq, R., Tesfamariam, S., A fuzzy Bayesian belief network for safety assessment of oil and gas pipelines, Structure and Infrastructure Engineering, 12 (8), pp. 874 889, 2016.

Katipamula, S., Brambley, M.R., Methods for fault detection, diagnostics, and prognostics for building systems - A review, Part I, HVAC and R Research, 11 (1), pp. 3-25, 2005.

Kim, C.-W., Morita, T., Oshima, Y., Sugiura, K., A Bayesian approach for vibration-based long-term bridge monitoring to consider environmental and operational changes, Smart Structures and Systems, 15 (2), pp. 395-408, 2015.

Kulak, O., Durmusoglu, B., Kahraman, C., Fuzzy multiattribute equipment selection based on information axiom, Journal of Materials Processing Technology 169, 337-345, 2005.

Network Rail, Delivering a better railway for a better Britain: our plans for 2014-2019, Network Rail Kings Place 90 York Way, London, N1 9AG, 2014.

Pipinato, A., Patton, R., Chapter 19 - Railway bridges, Innovative Bridge Design Handbook Construction, Rehabilitation and Maintenance, Pages 509-527, 2016.

Rafiq, M.I., Chryssanthopoulos, M.K., et al., Bridge condition modelling and prediction using dynamic Bayesian belief networks, Structure and Infrastructure Engineering, 11 (1), pp. 38-50, 2015.

Sanayei, M., Khaloo, A., Gul, M., Necati Catbas, F., Automated finite element model updating of a scale bridge model using measured static and modal test data, Engineering Structures, 102, pp. 66-79, 2015.

Teughels, A., Maeck, J., De Roeck, G., Damage assessment by FE model updating using damage functions, Computers and Structures, 80 (25), pp. 1869-1879, 2002.

Torfi, F., Farahani, R.Z., Rezapour, S. Fuzzy AHP to determine the relative weights of evaluation criteria and Fuzzy TOPSIS to rank the alternatives, Applied Soft Computing Journal, 10 (2), pp. 520-528, 2010.

Venkatasubramanian, V., Rengaswamy, R., Kavuri, S.N., Yin, $\mathrm{K}$., A review of process fault detection and diagnosis part III: Process history based methods, Computers and Chemical Engineering, 27 (3), pp. 327-346, 2003.

Wang, Y.-M., Elhag, T.M.S., On the normalization of interval and fuzzy weights, Fuzzy Sets and Systems, 157 (18), pp. 2456-2471, 2006.

Wang, L., Chan, T.H.T., Review of vibration-based fault detection and condition assessment of bridge structures using structural health monitoring, the Second Infrastructure Theme Postgraduate Conference, Queensland University, 2009.

Zhao, R., Govind, R., Defuzzification of fuzzy intervals, Fuzzy Sets and Systems, 43 (1), pp. 45-55, 1991.

Zhong, R., Zong, Z., Niu, J., Yuan, S., A damage prognosis method of girder structures based on wavelet neural networks, Mathematical Problems in Engineering, 2014, art. no. $130274,2014$. 